\title{
Controlled Self-organization on Germanium Using Focused Ion Beam (FIB): From Quasi-periodic Nanoripples to Well-ordered Periodic Nanostructures
}

Bhaveshkumar Kamaliya ${ }^{1}$, Vivek Garg ${ }^{1}$, Rakesh Mote $^{2}$, Mohammed Aslam ${ }^{2}$ and Jing Fu ${ }^{3}$

${ }^{1}$ IITB Monash Research Academy, Mumbai, Maharashtra, India, ${ }^{2}$ Indian Institute of Technology Bombay, Mumbai, Maharashtra, India, ${ }^{3}$ Monash University, Clayton, Victoria, Australia

In order to fabricate nanostructures, direct milling by the focused ion beam (FIB) is considered as a maskless and single-step fabrication technique, but it is a time-consuming process. In addition to direct written nanostructures, capacities of FIB can be harnessed for ion beam induced self-organization of surface [1-4] and nanoscale manipulation [5]. The focused ion beam induced self-organized nanostructures has an advantage over direct milling due to the low dose requirement. Thus, creating FIB induced self-organized nanostructures is a faster and preferred approach. The formation of nanoripples on germanium has been reported by self-organization of the surface atoms due to focused ion beam bombardment $[3,4]$. However, methodical control on the topography evolution of germanium surface during FIB irradiation has not been studied earlier. The present work demonstrates controlled realization and evolution of varied nanostructures such as aligned-nanoripples [1] and periodic nanostructures on the Ge surface by FIB induced self-organization.

The dual-beam focused ion beam (FIB)-scanning electron microscope (SEM) systems (Auriga® compact; Carl Zeiss \& Helios NanoLab 600, Thermo Fisher-FEI, USA) were used for the fabrication experiments. When beam overlap was varied from zero to negative, it was observed that the alignment of the nanoripples is strongly dependent on the beam overlap [1]. The Ga+ FIB with $30 \mathrm{KeV}$ energy and 100 pA was raster scanned on the germanium (100) surface. Parallel, perpendicular and random orientation of nanoripples with respect to the scanning direction has been realized by optimized beam overlap with a normal incidence of the focused ion beam. The false coloured scanning electron microscopy (SEM) images in Figure 1(a) and (b) show the alteration of the nanoripples alignment by changing the beam overlap (BO) from $0 \%$ (Figure 1(a)) to $-250 \%$ (Figure 1(b)) [1]. The nanoripples are found to exhibit broadband light absorptance of $95 \%$ in the visible range (Figure 1(d)) of the electromagnetic spectrum due to the enhanced light trapping and multiple reflections as represented in Figure 1(c) [1].

In order to achieve the periodic nanostructures from the quasi-periodic nanoripples, further dose optimization with negative beam overlap of FIB has been carried out. The optimised process parameters led to providing control over the self-organization of the circular nanoholes which are created by the dwelling of FIB at each pixel. Controlled and periodic nanostructures are found to be evolved from the array of circular nanoholes. Moreover, these nanostructures are protruding out of the surface, providing the capability of creating nanoscaled 3D structures. The beam current (i.e. beam size) and beam overlap (pixel size) define the feature size of the polygonal nanostructures, and less than $10 \mathrm{~nm}$ feature size can be achieved through the demonstrated method. The $\mathrm{Ga}^{+} \mathrm{FIB}$ was irradiated with $30 \mathrm{KeV}$ energy at a small current of $9.7 \mathrm{pA}$ to fabricate periodic self-organised nanostructures on the germanium surface. SEM images of the periodic and self-organized nanostructures are shown in Figure 2. Based on the beam overlap and beam current settings, varied morphologies of the protruding nanostructures are obtained such as corrugated (Figure 2(a)), nano-scaled mushrooms (Figure 2(b)) and nanoneedle-nanohole dual-structures (Figure 2(c)). Corrugated nanostructures are formed with a single pass scanning of FIB with $-400 \%$ beam overlap and total dwell time of $10 \mathrm{~ms}$. Nano-mushrooms and dual-structured nano-features are obtained 
due to slightly elongated FIB with $-600 \%$ beam overlap (Figure 2(b \& c)). Nano-mushrooms are produced with a total dwell time of $38 \mathrm{~ms}$ in 10 pass scanning, and dual-structures are produced with a total dwell time of $32 \mathrm{~ms}$ in 20 pass scanning. It should be noted that for the elongated FIB spot, change in the dwell time and the number of passes make huge morphology difference due to preferred self-organization. It is interesting that the FIB irradiation on the silicon surface with negative BO dominates direct milling and does not exhibit such self-organization as observed for the germanium surface [6]. Thus, theses selforganized structures are unique for the germanium surface with the optimized beam parameters. The broadband light absorptance spectra show $\sim 80 \%$ absorptance for the periodic nanostructures (Figure 2(d)). Corrugated nanostructures exhibit high absorptance for the longer wavelengths in the visible range of electromagnetic spectrum while dual-nanostructures show high absorptance for the shorter wavelengths in the visible range. The profile of the absorptance spectra for mushroom-shaped nanostructures is the same for the entire range of the visible spectrum.

Here, we present a novel approach of maskless fabrication and morphology control of self-organized nanostructures on the germanium surface by varying the beam overlap and scanning strategies. These nanostructures can be useful for applications such as efficient germanium-based photovoltaic devices, photonics, localized surface plasmon resonance-based sensing and to create hydrophobic surfaces for applications in the microfluidic devices [7].
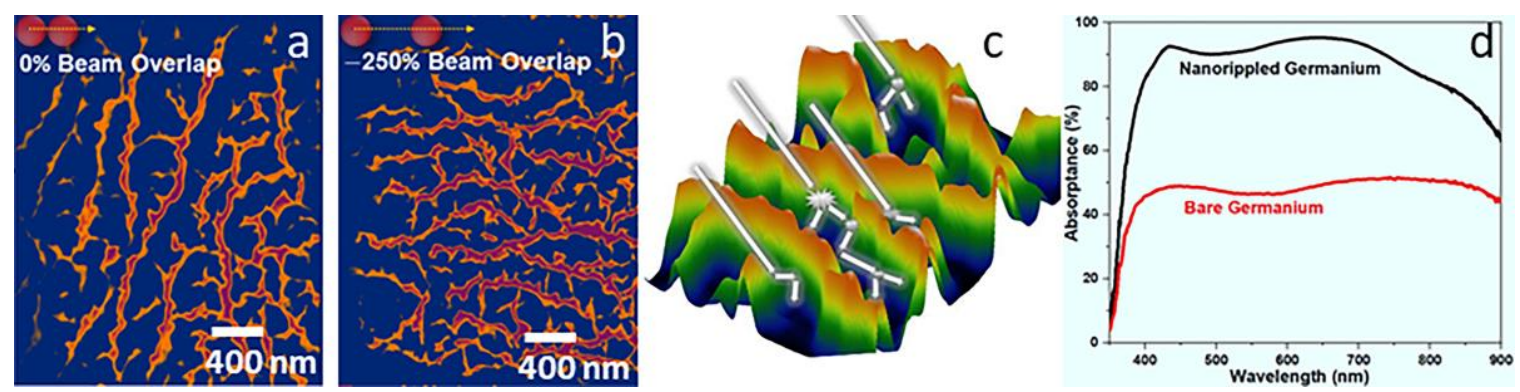

Figure 1. FIB induced germanium nanoripples: SEM images (false coloured) for (a) vertically and (b) horizontally quasi-periodic nanoripples on germanium surface, (c) mechanism of multiple reflections of light, (d) enhanced light absorption through nanoripples. Adapted from reference [1]. 

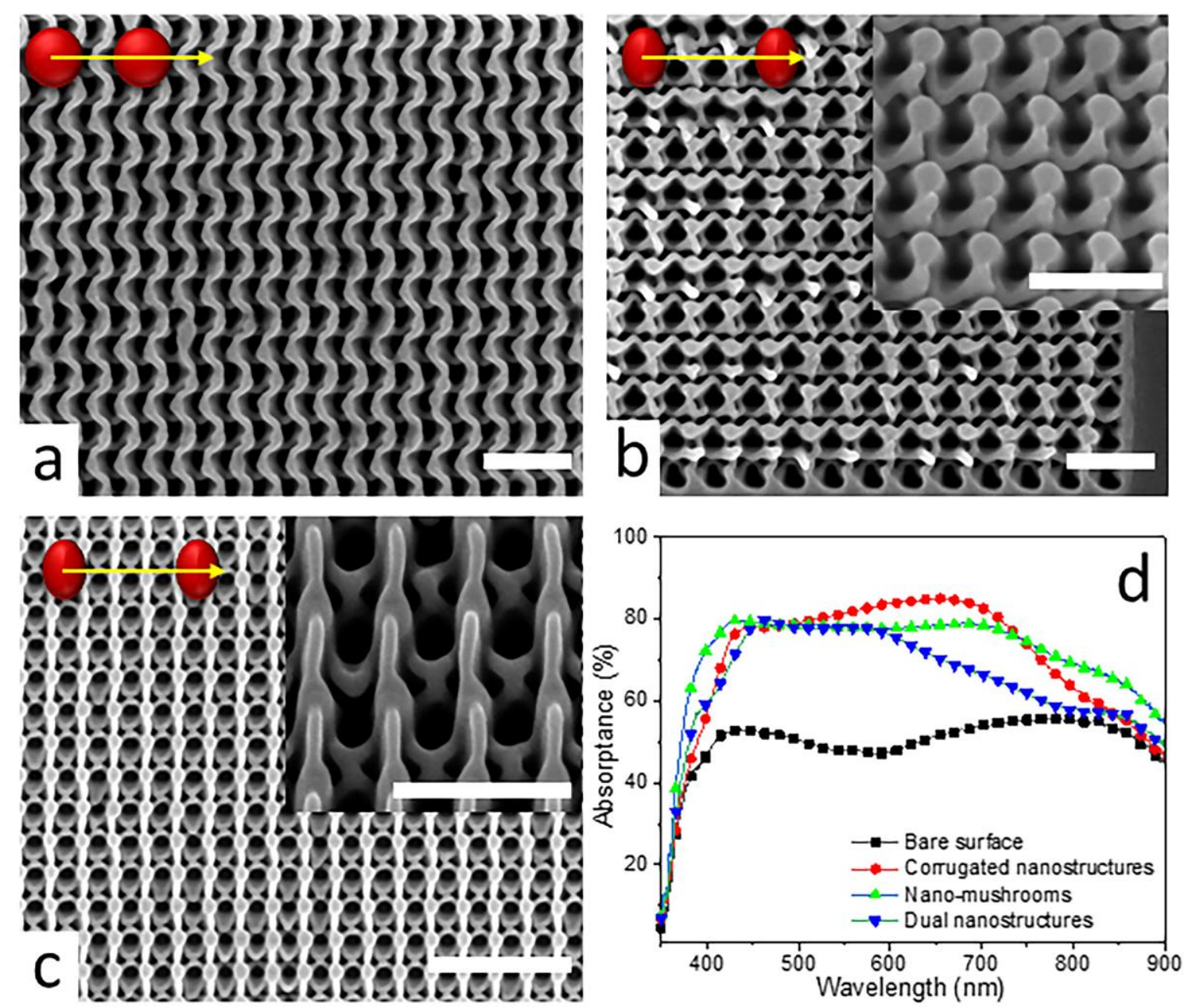

Figure 2. Periodic self-organization on germanium surface: (a) corrugated nanostructures produced with $-400 \%$ BO in 10 passes for total dwell time of $10 \mathrm{~ms}$, (b) nano-mushrooms (inset is tilted view at $30^{\circ}$ ) produced with elongated beam of $-600 \%$ BO in 10 passes for total dwell time of $38 \mathrm{~ms}$, (c) dualnanostructures on germanium resembling nano-holes connected with nano-needles (inset is tilted view at $30^{\circ}$ ) produced with elongated beam of $-600 \%$ BO in 20 passes for total dwell time of $32 \mathrm{~ms}$, and (d) absorptance spectra for the corresponding nanostructures in (a), (b) and (c). Scale bars in (a-c) represent $200 \mathrm{~nm}$.

\section{References}

1. B. Kamaliya, R. G. Mote, M. Aslam, and J. Fu, "Enhanced light trapping by focused ion beam (FIB) induced self-organized nanoripples on germanium (100) surface," APL Materials 6, 036106 (2018).

2. B. Kamaliya, R. G. Mote, M. Aslam, and J. Fu, "Improved Enhancement Factor for SERS using Broad Ion Beam Induced Self-organized Gold Nanocones," MRS Advances 4, 697 (2019).

3. R. M. Bradley and J. M. Harper, "Theory of ripple topography induced by ion bombardment," J. Vac. Sci. Technol. A 6, 2390 (1988).

4. W. Zhou, A. Cuenat, and M. J. Aziz, "Formation of self-organized nanostructures on Ge during focused ion beam sputtering," in Conference Series-Institute of Physics (Philadelphia; Institute of Physics; 1999, 2003), Vol. 180, pp. 625-628. 
5. V. Garg, B. Kamaliya, R. K. Singh, A. S. Panwar, J. Fu, and R. G. Mote, "Controlled Manipulation and Multiscale Modeling of Suspended Silicon Nanostructures under Site-Specific Ion Irradiation," ACS Applied Materials \& Interfaces 12, 6581-6589 (2020).

6. V. Garg, R. G. Mote, and J. Fu, "FIB fabrication of highly ordered vertical Gaussian pillar nanostructures on silicon," in 2017 IEEE 17th International Conference on Nanotechnology (IEEENANO) (2017), pp. 707-712.

7. The presented work was financially supported by IITB-Monash Research Academy, IRCC (Seed grant: Spons/ME/I14079-1/2014), IIT Bombay, NCPRE-II (funded through MNRE, Government of India) and Monash Engineering Seed Fund. This work was performed in part at IIT Bombay, the School of Chemistry (Monash University) and the Melbourne Centre for Nanofabrication (MCN) in the Victorian Node of the Australian National Fabrication Facility (ANFF). 